\title{
Vector and axial nucleon form factors: A duality constrained parameterization
}

\author{
A. Bodek ${ }^{\text {a }, ~ S . ~ A v v a k u m o v, ~ R . ~ B r a d f o r d, ~ H . ~ B u d d ~}$ \\ Department of Physics and Astronomy, University of Rochester, Rochester, NY 14627-0171, USA
}

Received: 12 October 2007 / Revised version: 21 November 2007 /

Published online: 15 December 2007 - ㄷ Springer-Verlag / Società Italiana di Fisica 2007

\begin{abstract}
We present new parameterizations of vector and axial nucleon form factors. We maintain an excellent descriptions of the form factors at low momentum transfers, where the spatial structure of the nucleon is important, and use the Nachtman scaling variable $\xi$ to relate elastic and inelastic form factors and impose quark-hadron duality constraints at high momentum transfers where the quark structure dominates. We use the new vector form factors to re-extract updated values of the axial form factor from neutrino experiments on deuterium. We obtain an updated world average value from $\nu_{\mu} \mathrm{d}$ and pion electroproduction experiments of $M_{\mathrm{A}}=1.014 \pm 0.014 \mathrm{GeV} / c^{2}$. Our parameterizations are useful in modeling neutrino interactions at low energies (e.g. for neutrino oscillations experiments). The predictions for high momentum transfers can be tested in the next generation electron and neutrino scattering experiments.
\end{abstract}

PACS. 13.40.Gp; 13.15.+g; 13.85.Dz; 14.20.Dh; 25.30.Bf; 25.30.Pt

\section{Introduction}

The nucleon vector and axial elastic form factors have been measured for more than 50 years in $e^{-} N$ and $\nu N$ scattering. At low $Q^{2}$, a reasonable description of the proton and neutron elastic form factors is given by the dipole approximation. The dipole approximation is a lowest-order attempt to incorporate the non-zero size of the proton into the form factors. The approximation assumes that the proton has a simple exponential spatial charge distribution, $\rho(r)=\rho_{0} e^{-r / r_{0}}$, where $r_{0}$ is the scale of the proton radius. Since the form factors are related in the non-relativistic limit to the Fourier transform of the charge and magnetic moment distribution, the above $\rho(r)$ yields the dipole form defined by:

$$
G_{D}^{\mathrm{V}, \mathrm{A}}\left(Q^{2}\right)=C^{\mathrm{V}, \mathrm{A}} /\left(1+\frac{Q^{2}}{M_{\mathrm{V}, \mathrm{A}}^{2}}\right)^{2}
$$

Here $C^{\mathrm{V}, \mathrm{A}}=\left(1, g_{\mathrm{A}}\right), g_{\mathrm{A}}=-1.267, M_{\mathrm{V}}^{2}=0.71(\mathrm{GeV} / c)^{2}$, and $M_{\mathrm{A}}$ is the axial mass.

Since $M_{\mathrm{A}}$ is not equal to $M_{\mathrm{V}}$, the distribution of electric and axial charge are different. However, the magnetic moment distributions were assumed to have the same spatial dependence as the charge distribution (i.e., form factor scaling). Recent measurements from Jefferson Lab show that the ratio of $\frac{\mu_{p} G_{E p}}{G_{M p}}$ falls at high $Q^{2}$ challenging the validity of form factor scaling [1] and resulting in new up-

\footnotetext{
a e-mail: bodek@pas.rochester.edu
}

dated parameterizations of the form factors [2], [3]. In this paper we present parameterizations that simultaneously satisfy constraints at low $Q^{2}$ where the spatial structure of the nucleon is important, and at high $Q^{2}$ where the quark structure is important. A violation of form-factor scaling is expected from quark-hadron duality.

We use our new vector form factors to re-extract updated values of the axial form factor from a re-analysis of previous neutrino scattering data on deuterium and present a new parameterization for the axial form factor within the framework of quark-hadron duality.

\section{New parametrization}

The new parameterizations presented in this paper are referred to as the duality based "BBBA07" parameterization. Our updated parameterizations feature the following:

1. Improved functional form that adds an additional $Q^{2}$ dependence using the Nachtman scaling variable $\xi$ to relate elastic and inelastic form factors. For elastic scattering $(x=1)$

$$
\xi^{p, n, N}=\frac{2}{\left(1+\sqrt{1+1 / \tau_{p, n, N}}\right)},
$$

where $\tau_{p, n, N}=Q^{2} / 4 M_{p, n, N}^{2}$. Here $M_{p, n, N}$ are the proton $\left(0.9383 \mathrm{GeV} / c^{2}\right)$, neutron $\left(0.9396 \mathrm{GeV} / c^{2}\right)$, and average nucleon mass (for proton, neutron, and axial form factors, respectively). 
2. Yield the same values as Arrington and Sick [4] for $Q^{2}<0.64(\mathrm{GeV} / c)^{2}$, while satisfying quark-hadron duality constraints at high- $Q^{2}$.

For vector form factors our fit functions are $A_{N}(\xi)$ (i.e. $\left.A_{E p}\left(\xi^{p}\right), A_{M p}\left(\xi^{p}\right), A_{E n}\left(\xi^{n}\right), A_{M n}\left(\xi^{n}\right)\right)$ multiplying an updated Kelly [3] type parameterization of one of the proton form factors. The Kelly parameterization is:

$$
G^{\text {Kelly }}\left(Q^{2}\right)=\frac{\sum_{k=0}^{m} a_{k} \tau_{p}^{k}}{1+\sum_{k=1}^{m+2} b_{k} \tau_{p}^{k}},
$$

where $a_{0}=1$ and $m=1$.

In our analysis, we use all the datasets used by Kelly [3], updated to include the recent BLAST [7] results, to fit $G_{E p}, G_{E n}, G_{M p} / \mu_{p}$, and $G_{M n} / \mu_{n}\left(\mu_{p}=2.7928, \mu_{n}=\right.$ $-1.9130)$. Our parameterization employs the published Kelly functional form to $G_{E p}^{\text {Kelly }}$, and an updated set of parameters for $G_{M P}^{\text {Kelly-upd }}\left(Q^{2}\right)$. The parameters used for $G_{E p}^{\text {Kelly }}$ and $G_{M p}^{\text {Kelly-upd }}$ are listed in Table 1 , and $A_{N}(\xi)$ is given by

$$
\begin{aligned}
A_{N}(\xi) & =\sum_{j=1}^{n} P_{j}(\xi) \\
P_{j}(\xi) & =p_{j} \prod_{k=1, k \neq j}^{n} \frac{\xi-\xi_{k}}{\xi_{j}-\xi_{k}} .
\end{aligned}
$$

Each $P_{j}$ is a LaGrange polynomial in $\xi$. The $\xi_{j}$ are equidistant "nodes" on an interval $[0,1]$, and $p_{j}$ are the fit parameters that have an additional property $A_{N}\left(\xi_{j}\right)=p_{j}$. The functional form $A_{N}(\xi)$ (for $G_{E p}, G_{M p}, G_{E n}$, and $\left.G_{M n}\right)$ is used with seven $p_{j}$ parameters at $\xi_{j}=0,1 / 6$, $1 / 3,1 / 2,2 / 3,5 / 6$, and 1.0 . In the fitting procedure described below, the parameters of $A_{N}(\xi)$ are constrained to give the same vector form factors as the recent low $Q^{2}$ fit of Arrington and Sick [4] for $Q^{2}<0.64(\mathrm{GeV} / c)^{2}$ (as that analysis includes coulombs corrections which modify $G_{E p}$, and two photon exchange corrections which modify $G_{M p}$ and $\left.G_{M n}\right)$. Since the published form factor data do not have these corrections, this constraint is implemented by including additional " $f a k e$ " data points for $Q^{2}<0.64(\mathrm{GeV} / c)^{2}$.

Our fits to the form factors are:

$$
\begin{aligned}
G_{M p}\left(Q^{2}\right) / \mu_{p} & =A_{M p}\left(\xi^{p}\right) G_{M p}^{\text {Kelly-upd }}\left(Q^{2}\right) \\
G_{E p}\left(Q^{2}\right) & =A_{E p}\left(\xi^{p}\right) G_{E p}^{\text {Kelly }}\left(Q^{2}\right) \\
G_{M n}\left(Q^{2}\right) / \mu_{n} & =A_{M n}^{25,43}\left(\xi^{n}\right) G_{M p}\left(Q^{2}\right) / \mu_{p} \\
G_{E n}\left(Q^{2}\right) & =A_{E n}^{25,43}\left(\xi^{n}\right) G_{E p}\left(Q^{2}\right)\left(\frac{a \tau_{n}}{1+b \tau_{n}}\right),
\end{aligned}
$$

where we use our updated parameters in the Kelly parameterizations. For $G_{E n}$ the parameters $a=1.7$ and $b=3.3$ are the same as in the Galster [9-11] parametrization and ensure that $\mathrm{d} G_{E n} / \mathrm{d} Q^{2}$ at for $Q^{2}=0$ is in agreement with measurements.

For convenience, we also provide fits for the form factors $G_{E p}$ and $G_{M p} / \mu_{p}$ that give very close to the same values,
Table 1. Parameters for $G_{E p}^{\mathrm{Kelly}}$ and $G_{M p}^{\mathrm{Kelly}-u p d}$. Our parameterization employs the as-published Kelly parameterization to $G_{E p}^{\text {Kelly }}$ and an updated set of parameters for $G_{M p}^{\text {Kelly-upd }}\left(Q^{2}\right)$ that includes the recent BLAST [7] results

\begin{tabular}{lcccrc}
\hline & $a_{1}$ & $b_{1}$ & $b_{2}$ & $b_{3}$ & $\chi^{2} / n d f$ \\
\hline$G_{\text {Ep }}^{\text {Kelly }}$ & -0.24 & 10.98 & 12.82 & 21.97 & 0.78 \\
$G_{M p}^{\text {Kelly-upd }}$ & 0.1717 & 11.26 & 19.32 & 8.33 & 1.03 \\
\hline
\end{tabular}

but use the dipole form instead:

$$
\begin{aligned}
G_{E p}\left(Q^{2}\right) & =A_{E p \text {-dipole }}\left(\xi^{p}\right) G_{D}^{\mathrm{V}}\left(Q^{2}\right) \\
G_{M p}\left(Q^{2}\right) / \mu_{p} & =A_{M p \text {-dipole }}\left(\xi^{p}\right) G_{D}^{\mathrm{V}}\left(Q^{2}\right) .
\end{aligned}
$$

The values $A(\xi)=p_{1}$ at $\xi_{1}=0\left(Q^{2}=0\right)$ for $G_{M p}, G_{E p}$, $G_{E n}, G_{M n}$ are set to to 1.0. The value $A(\xi)=p_{7}$ at $\xi_{j}=1$ $\left(Q^{2} \rightarrow \infty\right)$ for $G_{M p}$ and $G_{E p}$ is set to 1.0.

The value $A(\xi)=p_{j}$ at $\xi_{j}=1$ for $G_{M n}$ and $G_{E n}$ are fixed by constraints from quark-hadron duality. Quark-hadron duality implies that the ratio of neutron and proton magnetic form factors should be the same as the ratio of the corresponding inelastic structure functions $\frac{F_{2 n}}{F_{2 p}}$ in the $\xi=1$ limit. (Here $F_{2}=\xi \sum_{i} e_{i}^{2} q_{i}(\xi)$ )

$$
\frac{G_{M n}^{2}}{G_{M p}^{2}}=\frac{F_{2 n}}{F_{2 p}}=\frac{1+4 \frac{d}{u}}{4+\frac{d}{u}}=\left(\frac{\mu_{n}^{2}}{\mu_{p}^{2}}\right) A_{M n}^{2}(\xi=1) .
$$

We ran fits with two different values of $\frac{d}{u}$ at the $\xi=1$ limit: $\frac{d}{u}=0$ and 0.2 (corresponding to $\frac{F_{2 n}}{F_{2 p}}=0.25$ and $0.4286)$. The fit utilizing $\frac{d}{u}=0$ is $A_{M n}^{25}$, and the fit utilizing $\frac{d}{u}=0.2$ is $A_{M n}^{43}$. The final parameters for both cases of $\frac{d}{u}$, are given in Table 2 (or download computer code [31]). The difference between these two sets is indicative of the theoretical error of our parameterization.

Our parameterizations are within the error band of recent theoretical fits based in dispersion relations [12]. Since our fits are constrained to give the same vector form factors as the recent low $Q^{2}$ fit of Arrington and Sick [4] for $Q^{2}<0.64(\mathrm{GeV} / c)^{2}$, they are in agreement with the experimental measurements of the proton and neutron $\mathrm{rms}$ radii. (Note that as discussed in reference [13], the nucleon $\mathrm{rms}$ radius should be determined from fitting a polynomial of second order to the low $Q^{2}$ form factors. The commonly used polynomial of first order yields radius values which are too small).

The value $A(\xi)=p_{j}$ at $\xi_{j}=1$ for $G_{E n}$ is set by another duality-motivated constraint. $R$ is defined as the ratio of deep-inelastic longitudinal and transverse structure functions. For inelastic scattering, as $Q^{2} \rightarrow \infty, R_{n}=R_{p}$. If we assume quark-hadron duality, the same should be true for the elastic form factors at $\xi=1\left(Q^{2} \rightarrow \infty\right)$ limit:

$$
\begin{gathered}
R_{n}\left(x=1 ; Q^{2}\right)=\frac{4 M_{n}^{2}}{Q^{2}}\left(\frac{G_{E n}^{2}}{G_{M n}^{2}}\right) \\
G_{E n}^{2} / G_{M n}^{2}=G_{E p}^{2} / G_{M p}^{2} .
\end{gathered}
$$


Table 2. Fit parameters for $A_{N}(\xi)$, the LaGrange portion of the new parameterization. Note $A_{M n}^{25}, A_{E n}^{25}$, and $A_{F A}^{25}$ are constrained to have $\frac{d}{u}=0$ at $\xi=1$, and $A_{M n}^{43}, A_{E n}^{43}$, are constrained to have $\frac{d}{u}=0.2$

\begin{tabular}{|c|c|c|c|c|c|c|c|}
\hline$\xi, Q^{2}$ & $\begin{array}{l}p_{1} \\
0,0\end{array}$ & $\begin{array}{c}p_{2} \\
0.167,0.029\end{array}$ & $\begin{array}{c}p_{3} \\
0.333,0.147\end{array}$ & $\begin{array}{c}p_{4} \\
0.500,0.440\end{array}$ & $\begin{array}{c}p_{5} \\
0.667,1.174\end{array}$ & $\begin{array}{c}p_{6} \\
0.833,3.668\end{array}$ & $\begin{array}{l}p_{7} \\
1.0, \infty\end{array}$ \\
\hline$A_{E p}$ & 1. & 0.9927 & 0.9898 & 0.9975 & 0.9812 & 0.9340 & 1. \\
\hline$A_{E p \text {-dipole }}$ & 1. & 0.9839 & 0.9632 & 0.9748 & 0.9136 & 0.5447 & -0.2682 \\
\hline$A_{M p \text {-dipole }}$ & 1. & 0.9916 & 0.9771 & 0.9801 & 1.0321 & 1.0429 & 0.5084 \\
\hline$A_{M n}^{25}$ & 1. & 0.9958 & 0.9877 & 1.0193 & 1.0350 & 0.9164 & 0.7300 \\
\hline$A_{E n}^{25}$ & 1. & 1.1011 & 1.1392 & 1.0203 & 1.1093 & 1.5429 & 0.9706 \\
\hline$A_{E n}^{43}$ & 1. & 1.1019 & 1.1387 & 1.0234 & 1.1046 & 1.5395 & 1.2708 \\
\hline$A_{F A}^{25 \text {-dipole }}$ & 1.0000 & 0.9207 & 0.9795 & 1.0480 & 1.0516 & 1.2874 & 0.7707 \\
\hline
\end{tabular}
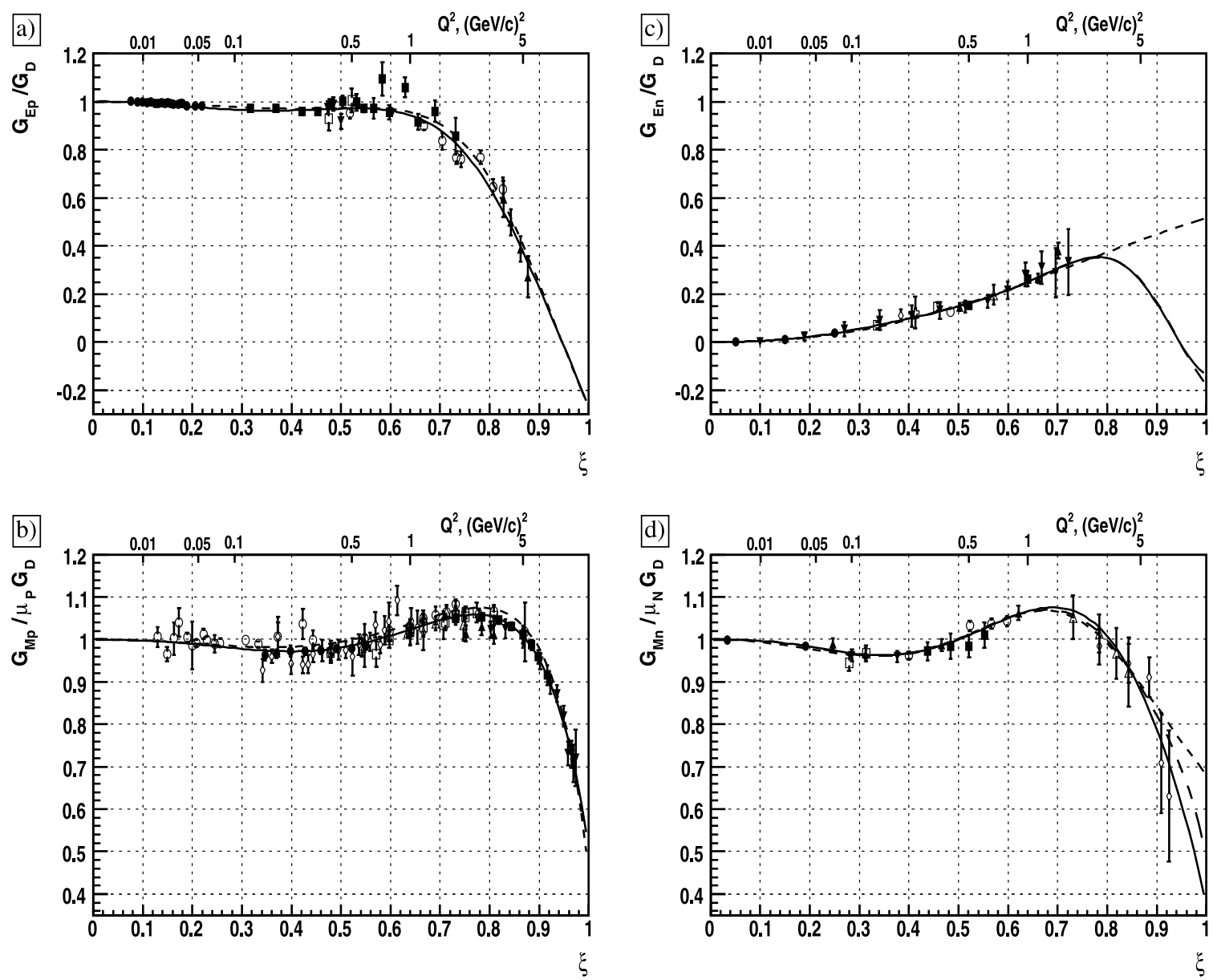

Fig. 1. Ratios of $G_{E p}(\mathbf{a}), G_{M p} / \mu_{p}(\mathbf{b}), G_{E n}(\mathbf{c})$ and $G_{M n} / \mu_{n}(\mathbf{d})$ to $G_{D}$. The short-dashed line in each plot is the old Kelly parameterizations (old Galster for $G_{E n}$ ). The solid line is our new BBBA07 25 parameterization for $\frac{d}{u}=0.0$, and the long-dashed line is BBBA07 43 for $\frac{d}{u}=0.2$. The values of $\xi$ and the corresponding values of $Q^{2}$ are shown on the bottom and top axis

In order to constrain the fits to $G_{E n}$ at high $Q^{2}$ we have assumed that the values of $\frac{G_{E n}^{2}}{G_{M n}^{2}}$ are the same as the measured $\frac{G_{E p}^{2}}{G_{M p}^{2}}$ for the three highest $Q^{2}$ data points for $G_{E p}$, and included these three "fake" data points in the $G_{E n}$ fits. In addition, the $R_{n}=R_{p}$ condition yields the following constraint at $\xi=1$ :

$$
A_{E n}^{25,43}(\xi=1)=P_{7}=\left(\frac{b}{a}\right)\left(\frac{1+4 \frac{d}{u}}{4+\frac{d}{u}}\right)^{1 / 2},
$$




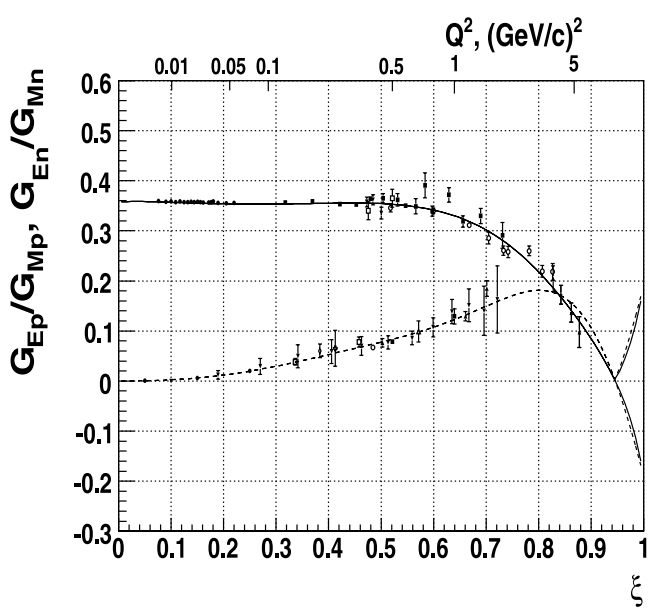

Fig. 2. The constraint used in fitting $G_{E n}$ stipulates that $G_{E n}^{2} / G_{M n}^{2}=G_{E p}^{2} / G_{M p}^{2}$ at high $\xi$. The solid line is $\frac{G_{E p}}{\left|G_{M p}\right|}$ and $\frac{\left|G_{E p}\right|}{\left|G_{M p}\right|}$, and the short-dashed line is $\frac{G_{E n}}{\left|G_{M n}\right|}$ and $\frac{\left|G_{E n}\right|}{\left|G_{M n}\right|}$

where $b / a=1.7 / 3.3$. As there are two parameter sets $A_{M n}^{25,43}(\xi)$, we have produced two parameter sets $A_{E n}^{25,43}$ as shown in Table 2.

The new form factors $G_{E p}, G_{M p} / \mu_{p}, G_{M n} / \mu_{n}$, and $G_{E n}$ are plotted in Fig. 1 as ratios to the dipole form $G_{D}^{\mathrm{V}}$.

As seen in Table $2, A_{N}(\xi)$ is not needed for $G_{M p}$ as it is very close to 1.0 . For $G_{E p}$ it yields a correction of $1 \%$ at low $Q^{2}$ (because it is required to agree with the fits of Arrington and Sick [4] which include two photon exchange and Coulomb corrections). For $G_{E n}$ and $G_{M n}$ it is used to impose quark-hadron duality asymptotic constraints. Figure 2 shows plots of the data and fits to $\frac{G_{E n}}{\left|G_{M n}\right|}$ and $\frac{G_{E p}}{\left|G_{M p}\right|}$ (for the $\frac{d}{u}=0$ at $\xi=1$ case).

\section{Re-extraction of axial form factor}

Using our updated $B B B A 2007_{25}$ form factors and an updated value $g_{\mathrm{A}}=-1.267$, we perform a complete reanalysis of published $\nu$ quasielastic [16-22] (QE) data on deuterium $\left(\nu_{\mu} n \rightarrow \mu^{-} p\right)$ using the procedure described in detail in $[2$, 14]. We extract new values of $M_{\mathrm{A}}$ with updated form factors $(\mathrm{FF})$ and also include radiative corrections $[5,6](\mathrm{RC})$. Although of lower statistical significance, for completeness we also include all available antineutrino data on hydrogen targets [23].

The average of the corrected measurements of $M_{\mathrm{A}}$ from Table 3 is $M_{\mathrm{A}}^{\text {deuterium }}=1.016 \pm 0.026 \mathrm{GeV} / c^{2}$. This is in agreement the average value of $M_{\mathrm{A}}^{\text {pion }}=1.014 \pm$ $0.016 \mathrm{GeV} / c^{2}$ extracted from pion electroproduction experiments after corrections for hadronic effects [15]. The average of the $\nu_{\mu}$ and electroproduction values is

$$
M_{\mathrm{A}}^{\text {world-average }}=1.014 \pm 0.014 \mathrm{GeV} / c^{2} .
$$

This precise $M_{\mathrm{A}}$ is smaller than the recent results (for $\left.Q^{2}>0.25(\mathrm{GeV} / c)^{2}\right)$ reported by MiniBoone [28] on a carbon target $\left(M_{\mathrm{A}}^{\text {carbon }}=1.25 \pm 0.12 \mathrm{GeV} / c^{2}\right)$ and by the $K 2 K[29]$ collaboration on oxygen $\left(M_{\mathrm{A}}^{\text {oxygen }}=1.20 \pm\right.$ $0.12 \mathrm{GeV} / c^{2}$ ). Both experiments use updated vector form factors. Although the collaborations attribute the larger $M_{\mathrm{A}}$ to nuclear effects, there are theoretical arguments that $M_{\mathrm{A}}$ in nuclear targets should be smaller [30] than (or the same [26]) as in deuterium. This $M_{\mathrm{A}}$ discrepancy is important for $\nu$ oscillations experiments since it affects the normalization (at high energies the QE cross section is approximately proportional to $M_{\mathrm{A}}$ ) and non-linearity of the QE cross section, which is relevant to the extraction of $\nu$ mass difference and mixing angle.

For deep-inelastic scattering, the vector and axial parts of $F_{2}$ are equal. Local quark-hadron duality at large $Q^{2} \mathrm{im}$ -

Table 3. $M_{\mathrm{A}}\left(\mathrm{GeV} / c^{2}\right)$ values published by $\nu_{\mu}$-deuterium experiments [16-22] and updated corrections $\Delta M_{\mathrm{A}}$ when re-extracted with updated BBBA2007 25 form factors, and $g_{a}=-1.267$. Also shown is updated $M_{\mathrm{A}}$ from $\bar{\nu}_{\mu}$ hydrogen $\rightarrow \mu^{-} n$ [23]

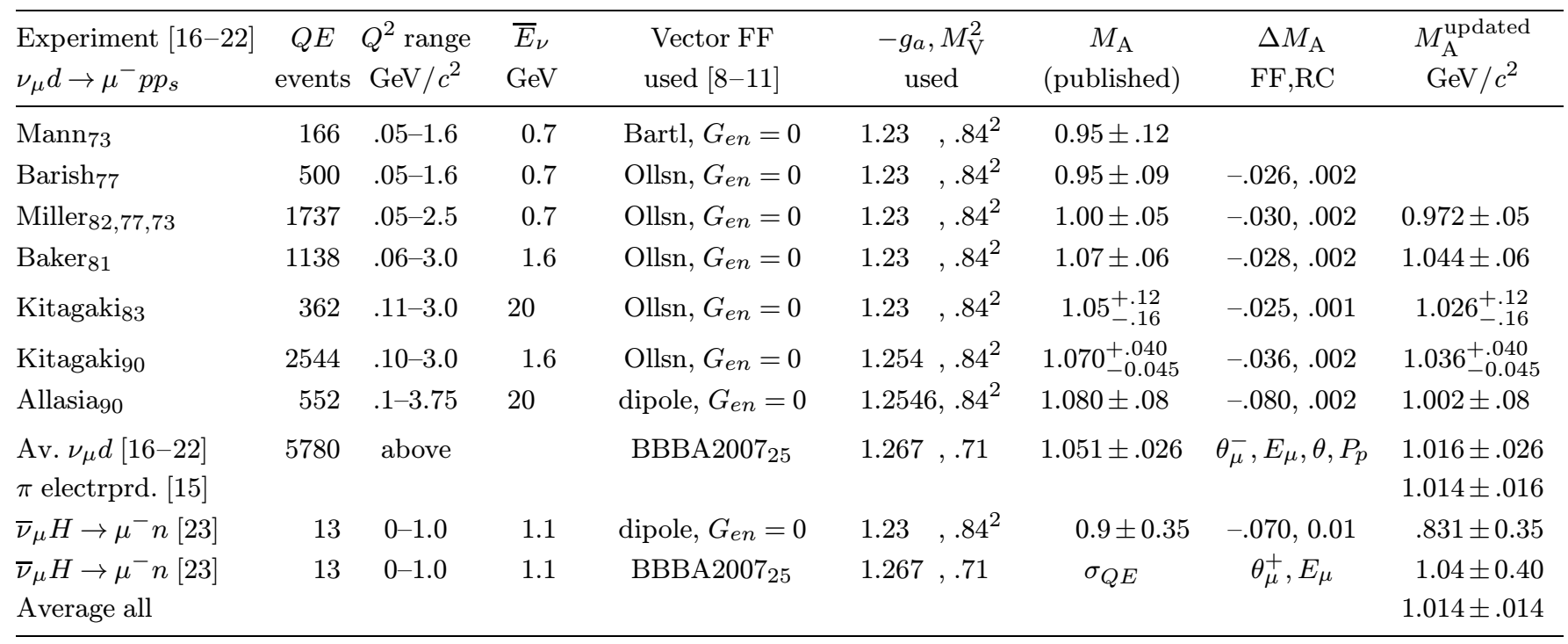



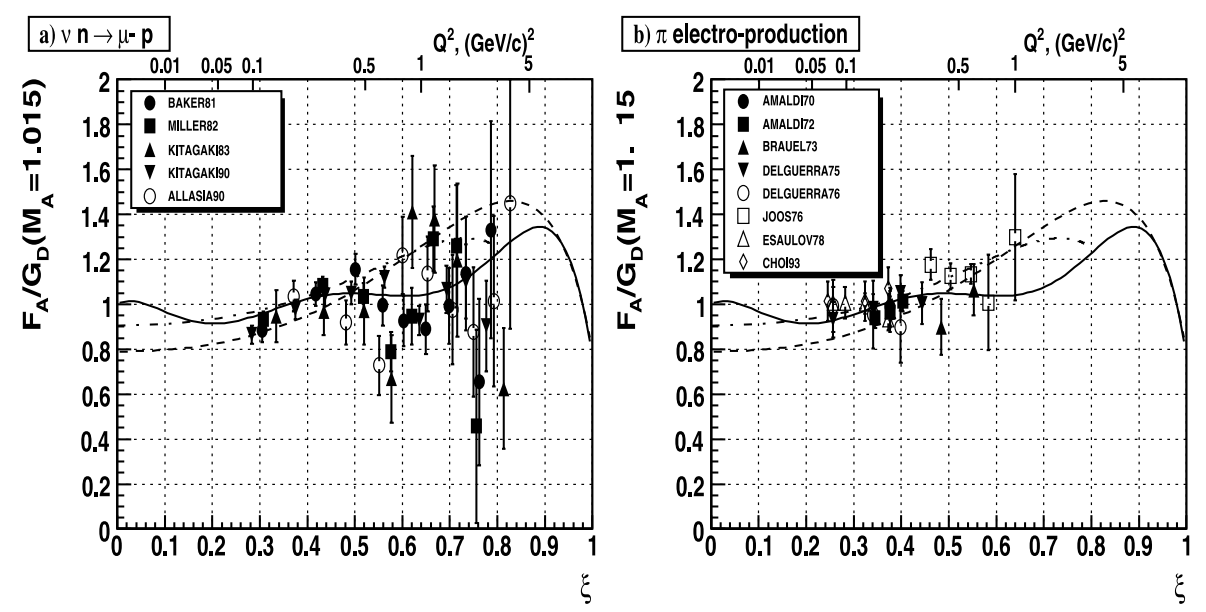

Fig. 3. (a) $F_{\mathrm{A}}\left(Q^{2}\right)$ re-extracted from neutrino-deuterium data divided by $G_{D}^{\mathrm{A}}\left(Q^{2}\right)[32]$. (b) $F_{\mathrm{A}}\left(Q^{2}\right)$ from pion electroproduction divided by $G_{D}^{\mathrm{A}}\left(Q^{2}\right)$ [32], corrected for for hadronic effects [15]. Solid line-duality based fit; Short-dashed line $-F_{\mathrm{A}}\left(Q^{2}\right){ }_{A 2}=V 2$. Dashed-dot line - constituent quark model [27]

plies that the axial and vector components of $F_{2}^{\text {elastic }}$ are also equal, which yields:

$$
\left[F_{\mathrm{A}}\left(Q^{2}\right)_{A 2=V 2}\right]^{2}=\left(G_{E}^{\mathrm{V}}\right)^{2}\left(Q^{2}\right)+\tau_{N}\left(G_{M}^{\mathrm{V}}\left(Q^{2}\right)\right)^{2} /\left(1+\tau_{N}\right),
$$

where

$$
\begin{aligned}
G_{E}^{\mathrm{V}}\left(Q^{2}\right) & =G_{E p}\left(Q^{2}\right)-G_{E n}\left(Q^{2}\right), \quad \text { and } \\
G_{M}^{\mathrm{V}}\left(Q^{2}\right) & =G_{M p}\left(Q^{2}\right)-G_{M n}\left(Q^{2}\right) .
\end{aligned}
$$

We extract values of $F_{\mathrm{A}}\left(Q^{2}\right)$ from the differential cross sections using the procedure of [14]. The overall normalization is set by the theoretical QE cross section [32]. We then do a duality based fit to $F_{\mathrm{A}}\left(Q^{2}\right)$ (including pion electroproduction data) of the form:

$$
F_{\mathrm{A}}\left(Q^{2}\right)=A_{F A}^{25}\left(\xi^{N}\right) G_{D}^{\mathrm{A}}\left(Q^{2}\right) .
$$

We impose the constraint $A_{F A}^{25}\left(\xi_{1}=0\right)=p_{1}=1.0$. We also constrain the fit by requiring that $A_{F A}^{25}\left(\xi^{N}\right)$ yield $F_{\mathrm{A}}\left(Q^{2}\right)=F_{\mathrm{A}}\left(Q^{2}\right)_{A 2=V 2}$ by including additional "fake" data points) for $\xi>0.9\left(Q^{2}>7.2(\mathrm{GeV} / c)^{2}\right)$.

Figure $3 \mathrm{a}$ shows $F_{\mathrm{A}}\left(Q^{2}\right)$ extracted from neutrinodeuterium experiments divided by $G_{D}^{\mathrm{A}}\left(Q^{2}\right)$ [32]. Figure $3 \mathrm{~b}$ shows $F_{\mathrm{A}}\left(Q^{2}\right)$ extracted from pion electroproduction experiments divided by $G_{D}^{\mathrm{A}}\left(Q^{2}\right)$ [32]. These pion electroproduction values can be directly compared to the neutrino results because they are multiplied by a factor $F_{\mathrm{A}}\left(Q^{2}, M_{\mathrm{A}}=\right.$ $\left.1.014 \mathrm{GeV} / c^{2}\right) / F_{\mathrm{A}}\left(Q^{2}, M_{\mathrm{A}}=1.069 \mathrm{GeV} / c^{2}\right)$ to correct for $\Delta M_{\mathrm{A}}=0.055 \mathrm{GeV} / \mathrm{c}^{2}$ originating from hadronic effects [15]. The solid line is our duality based fit. The short-dashed line is $F_{\mathrm{A}}\left(Q^{2}\right)_{A 2=V 2}$. The dashed-dot line is a constituentquark model [27] prediction.

\section{Conclusion}

In conclusion, our new parameterizations of vector and axial nucleon form factors use quark-hadron duality constraints at high momentum transfers, and maintain a very good descriptions of the form factors at low momentum transfers. Our new parameterizations are useful in modeling $\nu$ interactions for oscillations experiments. Our predictions [33] for $G_{E n}\left(Q^{2}\right)$ and $F_{\mathrm{A}}\left(Q^{2}\right)$ at high $\left(Q^{2}\right)$ can be tested in future $e-N$ and $\nu$-N experiments. at Jefferson Laboratory and at Fermilab (MINERvA) [34].

\section{References}

1. O. Gayou et al., Phys. Rev. Lett. 88, 092301 (2002)

2. R. Bradford, H. Budd, A. Bodek, J. Arrington, Nucl. Phys. B 159, 127 (2006)

3. J.J. Kelly, Phys. Rev. C 70, 068202 (2004)

4. J. Arrington, I. Sick, Phys. Rev. C 76, 035201 (2007)

5. A. De Rújula, R. Petronzio, A. Savoy-Navarro, Nucl. Phys. B 154, 394 (1979)

6. A. Bodek hep/ex-0709.4004

7. C.B. Crawford et al., Phys. Rev. Lett. 98, 052301 (2007)

8. M.G. Ollson et al., Phys. Rev. D 17, 2938 (1978)

9. S. Galster et al., Nucl. Phys. B 32, 221 (1971)

10. P.E. Bosted, Phys. Rev. C 51, 409 (1995)

11. B. Bartoli et al., Riv. Nuovo Cimento 2, 241 (1972)

12. M.A. Belushkin, H.-W. Hammer, U.-G. Meissner, Phys. Rev. C 75, 035202 (2007)

13. F. Borkowski, G.G. Simon, V.H. Walther, R.D. Wendling, Z. Phys. A 275, 29 (1975)

14. H. Budd, A. Bodek, J. Arrington, Nucl. Phys. Proc. Suppl. 139, 90 (2005)

15. V. Bernard, L. Elouadrhiri, U. Meissner, J. Phys. G 28, R1 (2002)

16. W.A. Mann et al., Phys. Rev. Lett. 16, 3103 (1973)

17. S.J. Barish et al., Phys. Rev. D 16, 3103 (1977)

18. N.J. Baker et al., Phys. Rev. D 23, 2499 (1981)

19. K.L. Miller et al., Phys. Rev. D 26, 537 (1982)

20. T. Kitagaki et al., Phys. Rev. D 28, 436 (1983)

21. T. Kitagaki et al., Phys. Rev. D 42, 1331 (1990)

22. D. Allasia et al., Nucl. Phys. B 343, 285 (1990)

23. G. Fanourakis et al., Phys. Rev. D 21, 562 (1980) 
24. M. Sajjad Athar, Shakeb Ahmad, S.K. Singh, Phys. Rev. D 75, 093003 (2007)

25. T. Leitner, L. Alvarez-Ruso, U. Mosel, Phys. Rev. C 73, $065502(2006)$

26. K. Tsushima, Hungchong Kim, K. Saito, Phys. Rev. C 70, 038501 (2004)

27. R.F. Wegenbrunn et al., Few Body Syst. 14, 411 (2003) [hep-ph/0212190]

28. A. Aguilar-Areval et al. (MiniBoone) hep-ex/0706.0926
29. R. Gran et al. (K2K), Phys. Rev. D 74, 052002 (2006)

30. S.K. Singh, E. Oset, Nucl. Phys. A 542, 587 (1992)

31. http://www.pas.rochester.edu/ bodek/FF/

32. Evaluated with $M_{\mathrm{A}}=1.015 \mathrm{GeV} / c^{2}$

33. Local duality may not hold for the elastic peak alone, as we assumed, and the analysis may require using the sum of elastic and first resonance combined.

34. http://minerva.fnal.gov/ 